\title{
Photodynamic Therapy and Bevacizumab Combination for Polypoidal Choroidal Vasculopathy Refractory to Intravitreal Ranibizumab
}

\author{
Zeynep Alkin, Abdullah Ozkaya, Sibel Ahmet, Okkes Baz, Mesut Togac, Irfan Perente, Muhittin Taskapili \\ Beyoglu Eye Training and Research Hospital, Istanbul, Turkey
}

\begin{abstract}
Objectives: The present study is an evaluation of outcomes of photodynamic therapy (PDT) combined with intravitreal injections of bevacizumab for polypoidal choroidal vasculopathy (PCV) that was nonresponding to ranibizumab injections. Methods: This retrospective study included 37 eyes of 37 patients with PCV. Principal outcome measurements used were mean change in best corrected visual acuity (BCVA) and central macular thickness (CMT), and regression rate of polypoidal lesions.

Results: Mean follow-up time was II.8 \pm 7.2 months. Mean BCVA decreased significantly from $0.69 \pm 0.42$ logMAR at baseline to $0.78 \pm 0.42 \log M A R$ at final visit $(p=0.01)$. BCVA improved in $9(24.3 \%)$ eyes, decreased in II $(29.7 \%)$, and remained stable in 17 (45.9\%) eyes. Mean CMT decreased from $352.2 \mu \mathrm{m}$ (range: I39-573 $\mu \mathrm{m}$ ) at baseline to 3 I8 8 I I $7.1 \mu \mathrm{m}$ (range: 136-585 $\mu \mathrm{m})$ at final visit $(p=0.26)$. Seventeen (45.9\%) eyes achieved dry macula, defined as absence of intraretinal or subretinal fluid on optical coherence tomography at 6 months. In addition, 25 eyes (67.5\%) showed complete regression of polypoidal lesions.

Conclusion: PDT combined with bevacizumab may be beneficial in resolution of exudation in eyes refractory to ranibizumab injections. VA improvement may be obtained in limited number of eyes.
\end{abstract}

Keywords: Bevacizumab, photodynamic therapy, polypoidal choroidal vasculopathy, ranibizumab.

\section{Introduction}

Polypoidal choroidal vasculopathy (PCV) is a disorder characterized by multiple, recurrent, serosanguinous detachments of the retinal pigment epithelium and neurosensory retina secondary to leakage and bleeding from choroidal vascular lesions. Characteristic feature of the disease is polyp-like, terminal, aneurysmal dilations, with or without identifiable branching choroidal vascular network, that are best detected on indocyanine green angiography (ICGA) (I, 2). Since fluorescein angiography findings of PCV can mimic those of occult choroidal neovascularization (CNV), visualization of abnormal polypoidal lesions with ICGA is required to differ- entiate PCV from CNV in neovascular age-related macular degeneration (nAMD) $(3,4)$. Recent findings have shown that optical coherence tomography (OCT) imaging may also be utilized to diagnose PCV with very high sensitivity and specificity (>90\%) (5).

PCV has been considered a variant of nAMD due to similarities in phenotypic features, but they differ in natural course and treatment response (6-8). Recent findings indicated that use of antivascular endothelial growth factor (anti-VEGF) agents resulted in improvement in vision of patients with PCV (9-12). In the Ranibizumab for Polypoidal Choroidal Vasculopathy (PEARL) study, visual acuity (VA)

Address for correspondence: Zeynep Alkin, MD. Beyoglu Eye Training and Research Hospital, Bereketzade Cami Sok., 34421 Beyoglu, Istanbul, Turkey

Phone: +90 21225159 00, E-mail: zeynepalkin@gmail.com

Submitted Date: October 18, 2016 Accepted Date: November 08, 2016 Available Online Date: November 15, 2016 ${ }^{\circ}$ Copyright 2016 by Beyoglu Eye Training and Research Hospital - Available online at www.beyoglueye.com 
improved in $23 \%$ of PCV eyes with monthly ranibizumab injections for a year; however, this result was not as robust as the $33 \%$ and $40 \%$ seen in the Minimally Classic/ Occult Trial of the Anti-VEGF Antibody Ranibizumab in the Treatment of Neovascular AMD (MARINA) and Anti-VEGF Antibody for the Treatment of Predominantly Classic Choroidal Neovascularization in AMD (ANCHOR) trials, respectively (I3-I5).

Although anti-VEGF agents have been shown to provide some benefit in reducing exudation in PCV eyes, they were found to be less effective than photodynamic therapy (PDT) in terms of decreasing abnormal choroidal vasculature and there were various visual outcomes $(16,17)$. The Endovascular Valve Edge-to-Edge Repair Study (EVEREST), a randomized controlled trial, evaluated the results of standard PDT, intravitreal ranibizumab (IVR), or combination of the 2 . EVEREST study demonstrated that all 3 groups showed VA gain, but rate of complete regression of polyps was higher in PDT combined with IVR and PDT groups than IVR monotherapy group ( $77.8 \%$ and $71.4 \%$ vs $28.6 \%$ ) (18).

Currently, main treatment decision for majority of PCV cases is based on whether to begin with antiangiogenic therapy or with PDT. Combining PDT with anti-VEGF agents can potentially create a synergistic effect that would help increase polyp regression rate, and reduce fluid leakage (19). When recurrent or residual PCV lesions seen on ICGA are associated with exudative fluid revealed on OCT during anti-VEGF therapy, there is still a question whether or not to continue anti-VEGF therapy or switch to PDT.

The purpose of this study was to evaluate results of standard PDT combined with intravitreal bevacizumab (IVB) in patients with PCV considered refractory to IVR, as indicated by OCT.

\section{Methods}

We retrospectively reviewed medical records of consecutive patients with PCV considered non-responsive to previous IVR treatment who were treated with standard verteporfin PDT and IVB (Avastin; Genentech, South San Francisco, CA, USA) $1.25 \mathrm{mg}$ combination therapy between February 2012 and January 2015.

All study conduct adhered to the tenets of the Declaration of Helsinki. Written informed consent was obtained from all patients before both intravitreal administrations and PDT.

Diagnosis of PCV was based on findings of lesions showing polyp-like, choroidal vessel dilatations with or without a branching vascular network (BVN) in middle and late phases of ICGA obtained using the Heidelberg Retina Angiograph (HRA-2; Heidelberg Engineering, Heidelberg, Germany).

Patients who had central serous chorioretinopathy, retinal vascular disease, any neovascular maculopathy, glaucoma, or history of intraocular surgery other than phacoemulsification, or who had follow-up of less than 6 months after PDT were excluded.

Initially, patients received 3 consecutive monthly ranibizumab injections and were then re-treated according to pro re nata treatment regimen: OCT evidence of intra- or subretinal fluid or new macular hemorrhage, or decrease in VA of more than 5 ETDRS letters.

Eyes without any reduction of subretinal and/or intraretinal fluid after $\geq 3$ IVR injections were offered PDT and IVB combination therapy. According to the treatment policy of our national health insurance, additional IVR injections after PDT are outside the scope of payment. As a result of lack of reimbursement for IVR, we used IVB in the combination therapy instead of IVR. PDT with verteporfin (Visudyne; Novartis, Basel, Switzerland) (intravenous injection of verteporfin $6 \mathrm{mg} / \mathrm{m} 2$ and laser irradiation at $689 \mathrm{~nm}$ wavelengths, 600 $\mathrm{mW} / \mathrm{cm} 2$ irradiance for 83 seconds) was administered within I week after IVB $1.25 \mathrm{mg} / 0.05 \mathrm{~mL}$ injection if there were no lesions near the optic disc and no evidence of high-pigment epithelial detachment. Linear diameter of polyps and surrounding abnormally dilated choroidal vessels was measured on ICGA. Laser spot size was then selected to cover entire lesion seen on ICGA by adding $1000 \mu \mathrm{m}$ to greatest linear diameter.

All patients had monthly follow-up ophthalmic examination that included measurement of BCVA via ETDRS chart, slit-lamp biomicroscopy, measurement of intraocular pressure via Goldmann applanation tonometer, 90 diopter lens fundus examination, and OCT imaging (Spectralis; Heidelberg Engineering, Heidelberg, Germany). Repeat ICGA after PDT was performed if clinical examination suggested persistence or recurrence of polypoidal lesions. Demographic data, number of injections, and duration of follow-up were also recorded.

Retreatment decision after IVB and PDT combination therapy was based on the following findings: IVB was injected if persistent subretinal or intraretinal fluid or hemorrhage was observed I month after last injection, and PDT was administered in combination with IVB injection if polypoidal lesions were identified on repeat ICGA with any fluid present on OCT at least 3 months after previous PDT.

Primary outcomes of this study were mean change in VA and central macular thickness (CMT) between baseline and last visit. Secondary outcomes included regression rate of polyp/BVN during the study period.

We converted the decimal VA measured into logarithm of the minimal angle of resolution (logMAR) VA for analysis. CMT, defined as mean thickness of the neurosensory retina in a central I mm diameter area, was computed using OCT mapping software provided with the device. 
Table I. Baseline characteristics of the patients

\section{Characteristics}

$\mathrm{n}=\mathbf{3 7}$

Age (mean $\pm S D)$ (range), years

$67.7 \pm 8.8(50-88)$

Gender (M/F)

$25 / 12$

Baseline logMAR BCVA (mean $\pm S D)$ (range)

Baseline CMT (mean \pm SD) (range), $\mu \mathrm{m}$

$0.69 \pm 0.42(0.1-1.3)$

$352.2(139-573)$

Number of IVR injections before combination

therapy (mean $\pm S D)$ (range)

$6.1 \pm 4.3(3-2 I)$

BCVA: best corrected visual acuity; CMT: central macular thickness; logMAR: logarithm of the minimal angle of resolution; M/F: male/female.

Statistical analysis was performed using Statistical Package for Social Sciences (SPSS) version 20 software (SPSS, Chicago, IL, USA). Continuous variables were analyzed using Wilcoxon signed-rank test for paired data. All values of $p$ are 2 -sided with appropriate significance of $p<0.05$.

\section{Results}

Thirty-seven eyes of 37 patients were included in this study. Of the total, $25(67.6 \%)$ of the patients were male, and 12 (32.4\%) were female. Patient age ranged from 50 to 88 years, with mean of $67.7 \pm 8.8$ years. Baseline characteristics of the patients are provided in Table I. Mean number of intravitreal injections was $6.1 \pm 4.3$ (range: $3-2 \mid$ )/ $15.6 \pm \mid 3$.I months before starting combination therapy compared with $2.7 \pm 2.6$ (range: I-I I)/II $8 \pm 7.2$ months after the combination theraPy $(\mathrm{P}<0.00 \mathrm{I})$. Ten eyes $(27 \%)$ received repeat PDT.

Mean \pm SD logMAR BCVA decreased from $0.69 \pm 0.42$ (range: $0.1-1.3$ ) at baseline to $0.61 \pm 0.4 \mathrm{I}$ (range: $0.1-\mathrm{I} .3$ ) at 6 months ( $p=0.95)$, but difference was not statistically significant. By 6 months, BCVA improved by $\geq 0$.I logMAR unit in 9 $(24.3 \%)$ eyes, decreased by $\geq 0.1$ logMAR unit in II (29.7\%), and remained stable in 17 (45.9\%) eyes.

The mean \pm SD CMT decreased from $352.2 \pm 93.6 \mu \mathrm{m}$ (range: 139-573 $\mu \mathrm{m}$ ) at baseline to $305.8 \pm 120.3 \mu \mathrm{m}$ (range: $136-580 \mu \mathrm{m})$ at last visit $(p=0.06)$, but difference was not statistically significant. Seventeen eyes (45.9\%) had dry macula at 6 months. Treatment results can be seen in Table 2 .

Complete polyp regression was found in 25 eyes (67.5\%). None of the patients developed new subretinal hemorrhage during the study period.

\section{Discussion}

The current study has shown that some visual and morphological recovery can be expected when PDT is combined with bevacizumab in eyes with PCV that had persistent activity despite previous anti-VEGF therapy.

Various studies have demonstrated that combined PDT and anti-VEGF treatment is more effective than an-
Table 2. Treatment results

\begin{tabular}{lc} 
Outcomes & $\mathbf{n}=\mathbf{3 7}$ \\
$\mathrm{BCVA}$ logMAR (mean $\pm \mathrm{SD})($ range) & $0.6 \mathrm{I} \pm 0.4 \mathrm{I}(0.1-1.3)$ \\
$\mathrm{CMT}($ mean $\pm \mathrm{SD})($ range), $\mu \mathrm{m}$ & $305.8 \pm 120.3$ \\
$(\mathrm{I} 36-580 \mu \mathrm{m})$ & \\
Number of IVB injections (mean $\pm S D)$ (range) & $2.7 \pm 2.6(\mathrm{I}-\mathrm{II})$ \\
Number of PDT sessions (mean $\pm S D)$ (range) & $\mathrm{I} .2 \pm 0.45(\mathrm{I}-2)$ \\
\hline
\end{tabular}

BCVA: best corrected visual acuity; CMT: central macular thickness; logMAR: logarithm of the minimal angle of resolution; PDT, photodynamic therapy.

ti-VEGF monotherapy, reporting improved vision, reduced incidence of subretinal hemorrhage, and reduced recurrence of polyps $(12,19,20)$ Romano et al. demonstrated that PDT and IVB combined therapy was able to achieve morphological stabilization of lesions in PCV through rapid decrease in macular thickness and regression in size of polypoidal vascular lesion (2I). A comparative study conducted by Gomi et al. indicated that patients in combined therapy group had significantly better visual outcomes than IVB monotherapy group despite similar polyp resolution rates at 12 months (22). Lai et al. suggested that anti-VEGF agents are effective in reducing leakage and resolving subretinal or intraretinal fluid, but ineffective for polyp regression (12). Results of various studies suggest that regression of polypoidal lesions may be attributed mainly to PDT alone. However, rapid resolution of fluids and improving VA may be primarily result of anti-VEGF therapy. Because the effect of anti-VEGF therapy on polypoidal lesions is limited, combined therapy may well be a treatment option when recurrent or persistent exudative change is seen after anti-VEGF treatments.

Unfortunately, effectiveness of combination therapy may decrease after previous unsuccessful therapy and possible development of resistance to the applied treatment. Tomita et al. demonstrated that PDT and IVR combination therapy provided greater VA gain in eyes that had no history of previous treatment (23). Eyes that had unsuccessful anti-VEGF therapy before combination treatment showed some improvement in VA, but mean change was not statistically significant at 12 months. They also found substantial decrease in exudative changes. The authors concluded that PDT combined with anti-VEGF therapy led to significant visual recovery in treatment-naïve eyes with PCV, but not in eyes already treated with PCV.

In the present study, VA outcomes were less favorable than morphological outcomes indicated by OCT. In our study, combination treatment provided additional beneficial effect in limited number of eyes. Although direct comparisons between studies should not be made, it is perhaps 
noteworthy that in our study, baseline VA was poorer than that of Tomita's study (23). In addition, other risk factors that have been reported such as larger lesion size, proximity to the fovea, persistence of exudation, and eventual atrophy or scar formation at the macula, may be responsible for poor VA outcomes. Due to retrospective design and non-uniform data records, we did not analyze the other factors above. We suggest that persistence of activity after previous anti-VEGF treatment and poor VA levels at baseline could be risk factor for VA outcomes after combination therapy.

Cho et al. reported that $75 \%$ of eyes with PCV previously treated with anti-VEGF agents showed complete resolution of polypoidal lesions after starting combination therapy with PDT and IVR/IVB (8). Despite stabilization of disease in most eyes, however, VA did not improve. The authors suggested that permanent photoreceptor damage from chronic edema resulted in lack of increase in VA. Polyp regression rate in present study was somewhat similar to that of the study by Cho et al. (67.5\% vs $75 \%)$.

Outcomes from the EVEREST study indicated that combining PDT with IVR resulted in fewer re-treatments with IVR over 5 months compared with IVR monotherapy (I8). In the current study, compared with 15.6-month period before starting combination therapy, the need for anti-VEGF injections during the II.8-month period after combination therapy was significantly reduced (6.I vs 2.7 injections).

Limitations of current study were retrospective design and relatively small sample size, and that follow-up time varied among patients.

To date, limited information is available on combined therapy for PCV that is refractory to previous anti-VEGF therapy. Of the 37 eyes analyzed in this study, 26 (70.2\%) had improved or demonstrated stable BCVA after the therapy. This was accompanied by an improvement in macular morphology as documented by OCT and ICGA. In conclusion, current study demonstrated beneficial short-term visual and morphological outcomes of PDT and IVB combination therapy for eyes with PCV that were nonresponding to previous IVR injections.

\section{Disclosures}

Financial Disclosure: This retrospective study was not supported by any company. None of the authors has financial or proprietary interests in any material or method mentioned. These data have not been previously published.

Peer-review: Externally peer-reviewed.

Conflict of Interest: None declared.

Authorship Contributions: Involved in design and conduct of the study (ZA, AO, SA, OB); preparation and review of the study (ZA, AO, IP, MT); data collection (ZA, AO, SA, OB, MT); and statistical analysis $(\mathrm{ZA})$.

\section{References}

I. Yannuzzi LA, Sorenson J, Spaide RF, Lipson B. Idiopathic polypoidal choroidal vasculopathy (IPCV). Retina 1990;10:1-8. Crossree

2. Spaide RF, Yannuzzi LA, Slakter JS, Sorenson J, Orlach DA. Indocyanine green videoangiography of idiopathic polypoidal choroidal vasculopathy. Retina 1995;15:100-10. Crossre

3. Lim LS, Mitchell P, Seddon JM, Holz FG, Wong TY. Age-related macular degeneration. Lancet 2012;379:1728-38. Crossret

4. Stangos AN, Gandhi JS, Nair-Sahni J, Heimann H, Pournaras CJ, Harding SP. Polypoidal choroidal vasculopathy masquerading as neovascular age-related macular degeneration refractory to ranibizumab. Am J Ophthalmol 2010;150:666-73. Crossre

5. De Salvo G, Vaz-Pereira S, Keane PA, Tufail A, Liew G. Sensitivity and specificity of spectral-domain optical coherence tomography in detecting idiopathic polypoidal choroidal vasculopathy. Am J Ophthalmol 2014;158:1228-38. Erossre

6. Laude A, Cackett PD, Vithana EN, Yeo IY, Wong D, Koh AH, et al. Polypoidal choroidal vasculopathy and neovascular age-related macular degeneration: same or different disease? Prog Retin Eye Res 2010;29:19-29. Erossre

7. Gomi F, Tano Y. Polypoidal choroidal vasculopathy and treatments. Curr Opin Ophthalmol 2008;19:208-12. Crossre

8. Cho M, Barbazetto IA, Freund KB. Refractory neovascular age-related macular degeneration secondary to polypoidal choroidal vasculopathy. Am J Ophthalmol 2009;148:70-8. Crossre

9. Cheng $\mathrm{CK}$, Peng $\mathrm{CH}$, Chang CK, Hu CC, Chen LJ. One-year outcomes of intravitreal bevacizumab (avastin) therapy for polypoidal choroidal vasculopathy. Retina 201 I;31:846-56. Crossret

10. Gomi F, Sawa M, Sakaguchi H, Tsujikawa M, Oshima Y, Kamei $M$, et al. Efficacy of intravitreal bevacizumab for polypoidal choroidal vasculopathy. $\mathrm{Br} J$ Ophthalmol 2008;92:70-3. Erossre

II. Kokame GT, Yeung L, Lai JC. Continuous anti-VEGF treatment with ranibizumab for polypoidal choroidal vasculopathy: 6-month results. Br J Ophthalmol 2010;94:297-301. Erossre

12. Lai TY, Chan WM, Liu DT, Luk FO, Lam DS. Intravitreal bevacizumab (Avastin) with or without photodynamic therapy for the treatment of polypoidal choroidal vasculopathy. $\mathrm{Br} \mathrm{J}$ Ophthalmol 2008;92:66I-6. Crossre

13. Kokame GT, Yeung L, Teramoto K, Lai JC, Wee R. Polypoidal choroidal vasculopathy exudation and hemorrhage: results of monthly ranibizumab therapy at one year. Ophthalmologica 2014;231:94-102. Erossre

14. Brown DM, Kaiser PK, Michels M, Soubrane G, Heier JS, Kim RY, et al. Ranibizumab versus verteporfin for neovascular age-related macular degeneration. N Engl J Med 2006;355:1432-44.

15. Rosenfeld PJ, Brown DM, Heier JS, Boyer DS, Kaiser PK, Chung $\mathrm{CY}$, et al. Ranibizumab for neovascular age-related macular degeneration. N Engl J Med 2006;355:14 I 9-3 I. Crossre

16. Rouvas AA, Papakostas TD, Ntouraki A, Douvali M, Vergados I, Ladas ID. Photodynamic therapy, ranibizumab, and ranibizum- 
ab with photodynamic therapy for the treatment of polypoidal choroidal vasculopathy. Retina 201 I;31:464-74. Crossre

17. Mitamura Y, Kitahashi M, Kubota-Taniai M, Yamamoto S. Comparison of intravitreal bevacizumab to photodynamic therapy for polypoidal choroidal vasculopathy: short-term results. Indian J Ophthalmol 2010;58:29I-6. Crossre

18. Koh A, Lee WK, Chen LJ, Chen SJ, Hashad Y, Kim H, et al. EVEREST study: efficacy and safety of verteporfin photodynamic therapy in combination with ranibizumab or alone versus ranibizumab monotherapy in patients with symptomatic macular polypoidal choroidal vasculopathy. Retina 2012;32: 1453-64.

19. Koh AH; Expert PCV Panel, Chen LJ, Chen SJ, Chen Y, Giridhar $\mathrm{A}$, lida $\mathrm{T}$, et al. Polypoidal choroidal vasculopathy: evidence-based guidelines for clinical diagnosis and treatment. Retina 2013;33:686-716. Erossret
20. Song MH, Ryu HW, Roh YJ. One-year results of intravitreal ranibizumab with or without photodynamic therapy for polypoidal choroidal vasculopathy. Ophthalmologica 201 1;226:1 19-26.

2I. Romano MR, Cipollone U, Semeraro F, Rinaldi M, Costagliola C. Combined photodynamic therapy and intravitreal bevacizumab for idiopathic polypoidal choroidal vasculopathy: oneyear follow-up. Clin Ophthalmol 2010;4:1237-4I. Crossre

22. Gomi F, Sawa M, Wakabayashi T, Sasamoto Y, Suzuki M, Tsujikawa M. Efficacy of intravitreal bevacizumab combined with photodynamic therapy for polypoidal choroidal vasculopathy. Am J Ophthalmol 2010;150:48-54. Erossre

23. Tomita K, Tsujikawa A, Yamashiro K, Ooto S, Tamura H, Otani $A$, et al. Treatment of polypoidal choroidal vasculopathy with photodynamic therapy combined with intravitreal injections of ranibizumab. Am J Ophthalmol 2012;153:68-80. Crossret 